\title{
Rapid and sensitive detection of Candidatus Liberibacter asiaticus by loop mediated isothermal amplification combined with a lateral flow dipstick
}

Luciano A Rigano ${ }^{1,5}$, Florencia Malamud ${ }^{1,6}$, Ingrid G Orce ${ }^{2}$, Maria P Filippone ${ }^{2}$, Maria R Marano ${ }^{3}$, Alexandre Morais do Amaral ${ }^{4}$, Atilio P Castagnaro ${ }^{2}$ and Adrian A Vojnov ${ }^{1 *}$

\begin{abstract}
Background: Citrus Huanglongbing (HLB) is the most devastating bacterial citrus disease worldwide. Three Candidatus Liberibacter species are associated with different forms of the disease: Candidatus Liberibacter asiaticus, Candidatus Liberibacter americanus and Candidatus Liberibacter africanus. Amongst them, Candidatus Liberibacter asiaticus is the most widespread and economically important. These Gram-negative bacterial plant pathogens are phloem-limited and vectored by citrus psyllids. The current management strategy of HLB is based on early and accurate detection of Candidatus Liberibacter asiaticus in both citrus plants and vector insects. Nowadays, real time PCR is the method of choice for this task, mainly because of its sensitivity and reliability. However, this methodology has several drawbacks, namely high equipment costs, the need for highly trained personnel, the time required to conduct the whole process, and the difficulty in carrying out the detection reactions in field conditions.

Results: A recent DNA amplification technique known as Loop Mediated Isothermal Amplification (LAMP) was adapted for the detection of Candidatus Liberibacter asiaticus. This methodology was combined with a Lateral Flow Dipstick (LFD) device for visual detection of the resulting amplicons, eliminating the need for gel electrophoresis. The assay was highly specific for the targeted bacterium. No cross-reaction was observed with DNA from any of the other phytopathogenic bacteria or fungi assayed. By serially diluting purified DNA from an infected plant, the sensitivity of the assay was found to be 10 picograms. This sensitivity level was proven to be similar to the values obtained running a real time PCR in parallel. This methodology was able to detect Candidatus Liberibacter asiaticus from different kinds of samples including infected citrus plants and psyllids.
\end{abstract}

Conclusions: Our results indicate that the methodology here reported constitutes a step forward in the development of new tools for the management, control and eradication of this destructive citrus disease. This system constitutes a potentially field-capable approach for the detection of the most relevant HLB-associated bacteria in plant material and psyllid vectors.

Keywords: Huanglongbing, Candidatus Liberibacter asiaticus, Diaphorina citri

\footnotetext{
* Correspondence: avojnov@fundacioncassara.org.ar

'Instituto de Ciencia y Tecnología Dr. Cesar Milstein, Fundación Pablo

Cassará, Consejo Nacional de Investigaciones Científicas y Técnicas

(CONICET), Ciudad de Buenos Aires, Argentina

Full list of author information is available at the end of the article
} 


\section{Background}

Citrus Huanglongbing (HLB), literally from the Chinese "Yellow Shoot Disease", is one of the most devastating diseases that threaten citrus production worldwide [1]. HLB is characterized by blotchy mottling with green areas on leaves. The infected shoots are stunted, and the branches gradually die as the disease progresses [2]. With the increase in disease severity, the yield is reduced and fruits quality is degraded. These affected fruit are smaller, lighter and highly acidic [2]. There are no curative procedures, and control of HLB consists of preventing trees from becoming infected and eradicating infected plants. Consequently, accurate and simple detection methods play a central role in reducing the incidence of HLB. The difficulty of correct diagnoses is partly because of the generic nature of HLB symptoms. The disease is sometimes misdiagnosed as nutrient deficiencies or other plant diseases [3].

Three fastidious $\alpha$-Proteobacteria species of Candidatus Liberibacter, namely Candidatus Liberibacter asiaticus (Las), Candidatus Liberibacter americanus (Lam) and Candidatus Liberibacter africanus (Laf) are associated with HLB $[1,2,4]$. These three bacteria are associated with different forms of the disease and have worldwide distribution. Las has been reported to be the most widespread, destructive, and economically important, being present in Asia, Brazil and North America $[1,2]$. Lam and Laf are found in Brazil and Southern Africa respectively $[1,3,5]$. These pathogens are transmitted by grafting and by the sap-sucking psyllids Diaphorina citri in America and Asia, and Trioza erytreae in South Africa [6]. Diaphorina citri is considered the most serious pest of citrus worldwide, due primarily to its role as vector of Las [6]. The insect is present in America and Asia, and it spreads rapidly in residential and commercial plantings through natural ways, but also by commercial transport of infected plant material [6,7]. Worldwide, control of the psyllid Diaphorina citri as a vector is a central milestone in HLB management [6]. Therefore detection of infected insects is critical in preventing the spread of the disease [7].

Currently, the major initial detection procedure for Las is visual inspection based on disease symptoms in trees. Samples that are suspected to be positive are sent to diagnostic laboratories for secondary analysis. Several methodologies have been developed to detect Las in these samples, including serologic assays, electron microscopy, biological assays, DNA probes, Loop Mediated Isothermal Amplification, PCR and real-time PCR [1,8-16]. Many of these methods have the drawback of being time-consuming and requiring complex facilities. In addition to some of these approaches, detection of the pathogen in infected plants or vectors remain problematic [3]. In recent years, diagnosis of HLB by real time PCR methodologies has gained popularity due to its sensitivity and reliability $[3,4,9,15]$, however real time PCR requires an expensive thermal cycler with a fluorescence detector, and highly trained personnel to perform assays and analyze data. These requirements reduce the suitability of real time PCR as an assay that can be performed "in field" (i.e., at the sampling site) or at border phytosanitary controls, places where complex facilities may not be available.

Loop-mediated isothermal amplification (LAMP) is a novel DNA amplification technique that amplifies DNA with high specificity, efficiency and rapidity under isothermal conditions [17]. LAMP is based on the principle of autocycling strand displacement DNA synthesis performed by the Bst DNA polymerase, for the detection of a specific DNA sequence [17]. The technique uses four to six primers that recognize six to eight regions of the target DNA and provides very high specificity $[17,18]$. Amplification can be carried out in a simple and inexpensive device like a water bath at temperatures between 60 to $65^{\circ} \mathrm{C}$. LAMP produces large amounts of DNA [17] and shows high tolerance to biological contaminants [19], thereby simplifying sample preparation. Although LAMP products can be detected by gel electrophoresis, this procedure reduces the suitability for field applications.

As mentioned above, a LAMP methodology for the detection of Las has been previously reported [11]. That work focused on the detection of the DNA sequence of the $t u f \mathrm{~B}-s e c \mathrm{E}-n u s \mathrm{G}-r p l \mathrm{KAJL}-r p o \mathrm{~B}$ gene cluster present in the microorganism. The analysis of the amplification products was done by gel electrophoresis, or dot-blotting of the amplification products on a nylon membrane followed by staining with Mupid Blue, methods that are not compatible with field applications.

On our study, we target a hypothetical protein-coding sequence present in the genome of Las for the detection of this pathogen. To overcome the limitations associated with the gel electrophoresis, we coupled the LAMP amplification with a Lateral Flow Dipstick (LFD), which permits an accurate and straightforward detection of LAMP amplicons, eliminating the need of complex equipment and data analysis [20,21]. By using both LAMP and LFD technologies, this work describes the development of a new molecular diagnostic tool for the detection of Las.

\section{Results and discussion}

In order to develop a successful HLB management strategy, methods for rapid detection of pathogens in the field are required. Such detection would allow early diagnosis of an infection focus before its spread. LAMP provides an ideal alternative for detection, as it requires a single incubation temperature and obviates the need for 
expensive thermal cyclers [17]. The combination of this isothermal DNA amplification technique with LFD devices has proven to be robust and successful in field-capable molecular diagnostics [20-22]. The recent sequencing of Las genome has uncovered new DNA sequences that can be used for pathogen detection through DNA amplification technologies [23]. Using an "in silico" approach, we found a hypothetical protein coding sequence, CLIBASIA_05175 [GenBank: ACT57606.1], which was predicted to be highly specific for Las. A BLASTn search [24] using the sequence of CLIBASIA_05175 as query, found homologs of this DNA sequence on Candidatus Liberibacter solanacearum, Candidatus Liberibacter americanus and Liberibacter crescens, however, no high level of sequence match was found between CLIBASIA_05175 and these sequences. An individual pairwise alignment between CLIBASIA_05175 and its BLASTn hits (Additional file 1: Figure S1) shows multiple mismatches on the primer binding regions, making unlikely a positive amplification with DNA from these other microorganisms. Accordingly, a DNA sample of Candidatus Liberibacter americanus did not produce positive amplification on the LAMP assay targeting CLIBASIA_05175 (Additional file 2: Figure S2).

Reactions were optimized to establish the best assay conditions. To determine the optimal temperature, the reaction mixture was incubated at 60,63 or $65^{\circ} \mathrm{C}$ for 60 minutes. With all tested temperatures, Las-LAMP products displayed the typical ladder-like pattern on gel electrophoresis with no amplification in the negative control lacking DNA (Figure 1). However, at 63 or $65^{\circ} \mathrm{C}$ the reaction was slightly more efficient than at $60^{\circ} \mathrm{C}$, with no apparent difference between the first two. The specificity of the amplification was confirmed by sequencing (Additional file 3: Figure S3). As a result of this experiment, the temperature chosen for the assay was $65^{\circ} \mathrm{C}$, as higher temperatures generally produce more stringent conditions for primer binding and greater amplification specificity [25]. We employed a thermal cycler, a water bath or an incubator to maintain the temperature necessary for the LAMP assay. The results indicated that all these devices were equally capable of producing efficient amplification (Additional file 4: Figure S4). Interestingly, a recent study shows that LAMP can be carried out using chemically driven heaters, a situation that could allow Las-LAMP amplifications in electricity-free locations [26].

Next we evaluated the effect of an improvement to the classic LAMP amplification, described previously [18]. Two additional primers named loop primers were added to the reaction mixture. The role of these oligonucleotides is to accelerate the reaction by providing more starting sites for the LAMP auto-cycling process. As shown in the Figure 1, the reaction containing loop primers and incubated at $65^{\circ} \mathrm{C}$ for 30 minutes performed

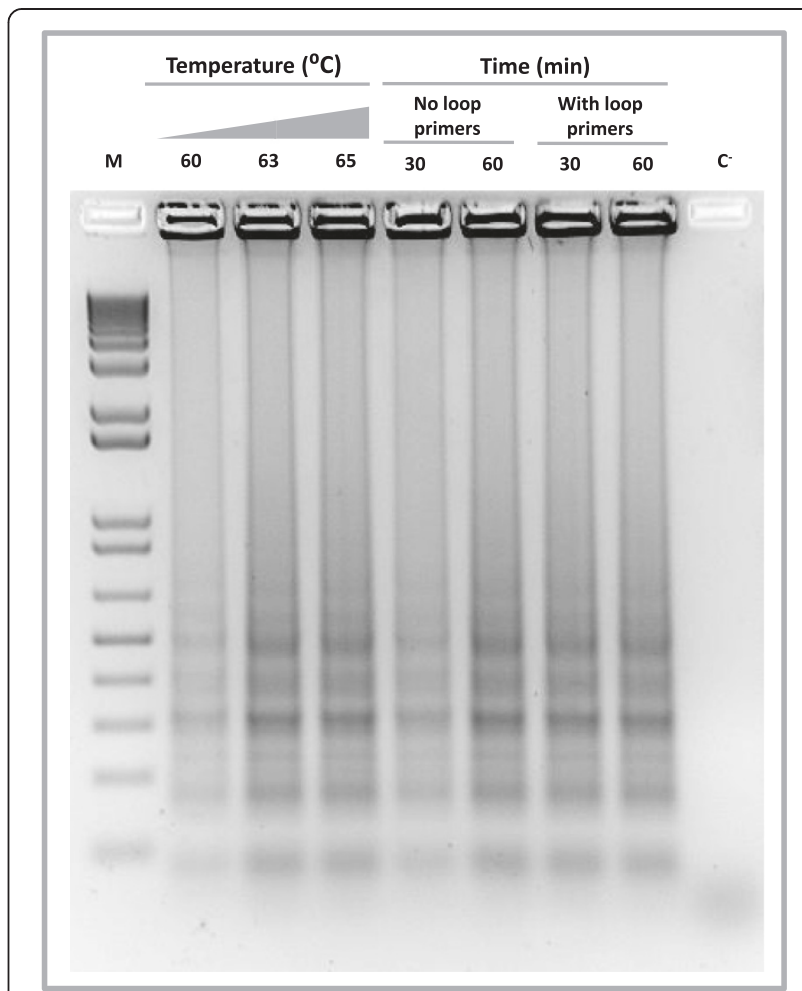

Figure 1 Las-LAMP reaction optimization. Several temperature, time and primer combinations were applied to Las-LAMP to determine optimal reaction conditions. An aliquot of $10 \mu \mathrm{l}$ of each Las-LAMP reaction was loaded into a 1.5\% agarose gel. After electrophoresis, the gel was stained with ethidium bromide. $C^{-}$: negative control without Template. M: 1 Kb plus DNA ladder (Invitrogen).

as well as the reaction without loop primers and incubated for 60 minutes. Therefore, the optimal reaction conditions that were used in all subsequent experiments consisted of incubation at $65^{\circ} \mathrm{C}$ for 30 minutes with the inclusion of loop primers to the amplification mix.

As previously mentioned, the analysis of LAMP amplicons by gel electrophoresis has several drawbacks, like the need of additional equipment, being a laborious and time consuming procedure, and requiring the use of the highly toxic ethidium bromide. These characteristics limit its use in field applications. To overcome these limitations, a generic lateral flow dipstick device (Milenia Biotec, Germany) was employed to detect the amplicons. This device detects biotin-labeled amplicons upon hybridization to a fluorescein isothiocyanate (FITC)labeled DNA probe complexed with a gold-labeled antiFITC antibody. The resulting triple complex moves by capillarity and is trapped by a biotin ligand at the test zone. As a result, the local gold concentration increases and a reddish-brown color line develops on the test zone during a positive reaction (Figure $2 \mathrm{~A}$ ).

When this methodology was used to detect Las-LAMP amplicons, we could distinguish two clear bands in the 


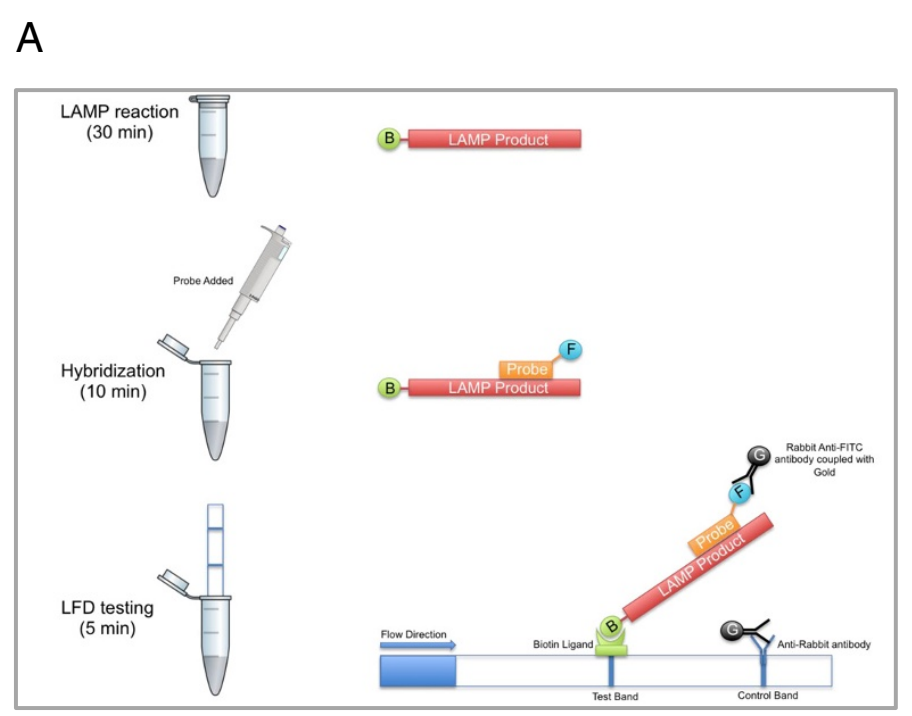

B

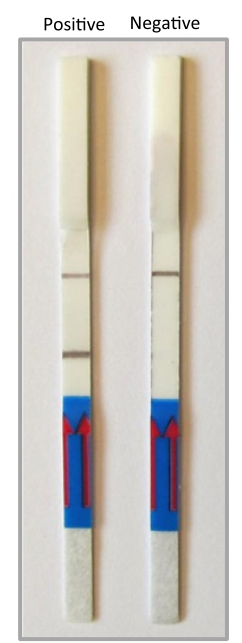

Figure 2 Lateral flow dipstick Las-LAMP evaluation. A. Lateral Flow Dipstick Las-LAMP procedure: LAMP reaction is performed using a biotinilated FIP primer. After 30 minutes of initial incubation at $65^{\circ} \mathrm{C}$, a specific FITC-labelled probe is added to the reaction mixture and incubated for another 10 minutes at the same temperature. This step produces a dual labeled LAMP product. Finally, detection buffer containing Rabbit Anti-FITC antibodies coupled with colloidal gold is mixed with the reaction mixture, and the LFD strip is inserted into the tube. In a positive reaction, double labeled LAMP products migrates with the buffer flow and are retained at the Test Band by a biotin ligand. The gold coupled Anti-FICT antibody binds to the FITC molecule at the probe and a dark band develops over the time. In the case of a negative reaction no products are generated and such process does not have place. An Anti-Rabbit antibody at the Control Band retains some of the unbound goldconjugated antibody and produces a Control Band that should be always visible. B. Evaluation of results using the Lateral Flow Dipstick device.

positive reaction. One of these bands was in the test zone and the other, which should be always present, was in the control zone. In contrast to the results with the positive reaction, in the negative control lacking DNA, only one band was visible and this was at the control zone (Figure 2B).

In order to determine the specificity of the Las-LAMP assay, purified DNA samples from several bacterial and fungal plant pathogens were evaluated. The results show that a positive reaction was obtained using DNA from plants infected with Las, but not with DNA from healthy plant material (Table 1, Additional file 5: Figure S5).

Negative results were obtained with DNA from other common citrus and plant pathogens, indicating a high level of specificity (Table 1). This specificity is likely due to the DNA region selected for amplification and also the nature of LAMP, which recognizes eight regions in the target DNA. LFD detection of the resulting amplicons adds another layer of specificity, because in order to be detected, the amplicons must hybridize specifically with the probe. Since genomic data is not available, and we have not analyzed samples of the related pathogen Candidatus Liberibacter africanus in this work, we can not exclude the possibility of a positive reaction with DNA from this pathogen.

The Las-LAMP assay sensitivity was determined using serial dilutions of total purified DNA from a Las positive plant. The same samples were evaluated in parallel by previously described real time PCR procedure [3] in order to compare sensitivities of both methods. Both gel electrophoresis and LFD detection of Las-LAMP amplicons showed the same detection limit of 10 picograms of DNA (Table 2, Additional file 5: Figure S5). Interestingly, this

Table 1 Specificity of the Las-LAMP assay

\begin{tabular}{|c|c|c|c|}
\hline \multirow[t]{2}{*}{ Species } & \multirow[t]{2}{*}{ Strain } & \multicolumn{2}{|c|}{ Detection method } \\
\hline & & Gel & LFD \\
\hline Candidatus Liberibacter asiaticus & * & + & + \\
\hline Xylella fastidiosa & $9 a 5 c$ & - & - \\
\hline Xanthomonas citri subsp. citri & 306 & - & - \\
\hline Xanthomonas campestris pv. campestris & 8004 & - & - \\
\hline Xanthomonas campestris pv. vesicatoria & $85-10$ & - & - \\
\hline Pseudomonas syringae & DC3000 & - & - \\
\hline Botrytis cinerea & B-191 & - & - \\
\hline Phytophthora citricola & * & - & - \\
\hline Guignardia citricarpa & * & - & - \\
\hline Elsinoe fawcettii & * & - & - \\
\hline Healthy Orange & $\mathrm{N} / \mathrm{A}$ & - & - \\
\hline Healthy Citrus limon & $\mathrm{N} / \mathrm{A}$ & - & - \\
\hline Healthy Diaphorina citri & N/A & - & - \\
\hline
\end{tabular}

For each sample Las-LAMP reaction was performed in triplicate. Gel: gel electrophoresis. LFD: lateral flow dipstick. +: Positive reaction. -: Negative reaction. *Performed with DNA from an infected plant without symptoms of other disease. N/A: Not applicable. 
Table 2 Comparison between Las-LAMP and real time PCR assay sensitivity from DNA purified from a Candidatus Liberibacter asiaticus positive plant

\begin{tabular}{lccccccc}
\hline Detection method & \multicolumn{7}{c}{ Purified DNA from a Las positive citrus plant } \\
\cline { 2 - 7 } & $\mathbf{1 0 0} \mathbf{~ n g}$ & $\mathbf{1 0} \mathbf{~ n g}$ & $\mathbf{1} \mathbf{~ n g}$ & $\mathbf{1 0 0} \mathbf{~ p g}$ & $\mathbf{1 0} \mathbf{~ p g}$ & $\mathbf{1 ~ p g}$ & $\mathbf{1 0 0} \mathbf{~ f g}$ \\
\hline Las-LAMP Gel & + & + & + & + & + & + & - \\
Las-LAMP FLD & + & + & + & + & + & - \\
Real time PCR & + & + & + & + & - \\
\hline
\end{tabular}

For each dilution the Las-LAMP reaction was performed in triplicate. Gel: gel electrophoresis. LFD: lateral flow dipstick. +: Positive reaction. -: Negative reaction. Real time PCR have been scored as positive if amplification could be detected during the reaction time.

detection limit was similar to that of the real time PCR assay. These results demonstrate that the fast and straightforward detection alternative that we describe here is at least as sensitive as the more complex and expensive approach of real time PCR.

The ability of this technique to detect Las in the vector psyllid, Diaphorina citri was evaluated using a simple and fast sample preparation method (Figure 3A). Briefly, one Las-infected insect was homogenized by vortexing in presence of InstaGene resin $\left(\mathrm{BIORAD}^{\circ}\right)$, incubated at $56^{\circ} \mathrm{C}$ for 20 minutes to activate the resin chelating groups and then incubated for 8 minutes at $100^{\circ} \mathrm{C}$ in order to destroy cellular structures and release the nucleic acids. When $5 \mu \mathrm{L}$ of this lysate was added to the Las-LAMP reaction mixture a positive amplification was detected by gel electrophoresis (data not shown) and LFD (Figure 3B). No amplification was detected with a non-infected insect. The same fast sample preparation method was evaluated for the detection of Las in leaf samples, but it did not perform well (data not shown). We are not certain of the reasons for this difference, but it might be related to the low number of bacteria present in the leaves compared with the psyllids and the presence of more potential amplification inhibitors present in leaf material.

Positive amplification was achieved with several field samples of Las extracted for DNA, including infected trees and psyllids (Table 3) demonstrating the robustness of the assay. However, further studies with a more diverse set of field samples, including those from
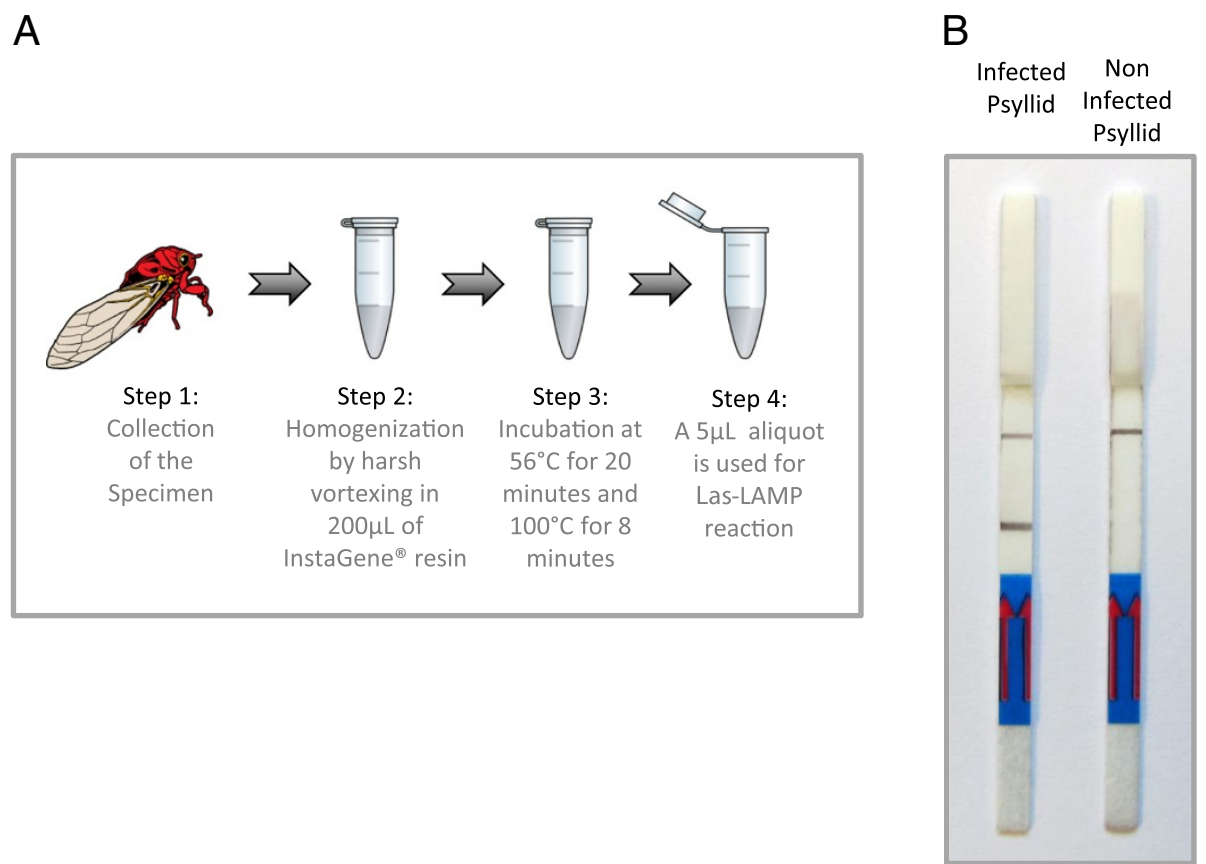

Figure 3 Fast detection of Las from Diaphorina citri. A. Fast psyllid sample preparation procedure: The specimen is grinded by harsh vortexing in $200 \mu \mathrm{L}$ of the PCR inhibitor chelator matrix InstaGene ${ }^{\oplus}\left(\mathrm{BIORAD}^{\mathrm{TM}}\right)$. After that, the sample is incubated at $56^{\circ} \mathrm{C}$ for 20 minutes to activate the matrix binding groups and subjected to a final incubation at $100^{\circ} \mathrm{C}$ for 8 minutes to disrupt cell material. A 5- $\mu \mathrm{L}$ aliquot is then used for Las-LAMP. B. Las-LAMP results from an infected psyllid (left) and an uninfected psyllid (right). On Figure 3A, the insect image is representative of a general flying insect for illustration purposes and it is not intended to represent an actual Diaphorina citri psyllid. 
Table 3 Samples of Candidatus Liberibacter asiaticus positive material used to evaluate the Las-LAMP assay

\begin{tabular}{|c|c|c|c|c|c|}
\hline \multirow[t]{2}{*}{ Species } & \multirow[t]{2}{*}{ Sample name } & \multicolumn{2}{|c|}{ Origin } & \multicolumn{2}{|c|}{ Detection method } \\
\hline & & Host & Country & Las-LAMP gel & Las-LAMP LFD \\
\hline Candidatus Liberibacter asiaticus & CLAS-12 & Orange & Brazil & + & + \\
\hline Candidatus Liberibacter asiaticus & CLAS-13 & Orange & Brazil & + & + \\
\hline Candidatus Liberibacter asiaticus & CLAS-14 & Orange & Brazil & + & + \\
\hline Candidatus Liberibacter asiaticus & CLAS-15 & Orange & Brazil & + & + \\
\hline Candidatus Liberibacter asiaticus & CLAS-16 & Orange & Brazil & + & + \\
\hline Candidatus Liberibacter asiaticus & CLAS-17 & Orange & Brazil & + & + \\
\hline Candidatus Liberibacter asiaticus & CLAS-18 & Orange & Brazil & + & + \\
\hline Candidatus Liberibacter asiaticus & CLAS-19 & Orange & Brazil & + & + \\
\hline Candidatus Liberibacter asiaticus & CLAS-20 & Orange & Brazil & + & + \\
\hline Candidatus Liberibacter asiaticus & CLAS-21 & Orange & Brazil & + & + \\
\hline Candidatus Liberibacter asiaticus & CHICHALAS-1 & Psyllid & Brazil & + & + \\
\hline Candidatus Liberibacter asiaticus & CHICHALAS-2 & Psyllid & Brazil & + & + \\
\hline Candidatus Liberibacter asiaticus & CHICHALAS-3 & Psyllid & Brazil & + & + \\
\hline Candidatus Liberibacter asiaticus & CHICHALAS-4 & Psyllid & Brazil & + & + \\
\hline Candidatus Liberibacter asiaticus & CHICHALAS-5 & Psyllid & Brazil & + & + \\
\hline Candidatus Liberibacter asiaticus & CHICHALAS-6 & Psyllid & Brazil & + & + \\
\hline Candidatus Liberibacter asiaticus & CHICHALAS-7 & Psyllid & Brazil & + & + \\
\hline Candidatus Liberibacter asiaticus & CHICHALAS-8 & Psyllid & Brazil & + & + \\
\hline Candidatus Liberibacter asiaticus & CHICHALAS-9 & Psyllid & Brazil & + & + \\
\hline Candidatus Liberibacter asiaticus & CHICHALAS-10 & Psyllid & Brazil & + & + \\
\hline Candidatus Liberibacter asiaticus & CHICHALAS-11 & Psyllid & Brazil & + & + \\
\hline
\end{tabular}

For each isolate the Las-LAMP reaction was performed in triplicate. Gel: gel electrophoresis. LFD: lateral flow dipstick. +: Positive reaction. -: Negative reaction.

worldwide locations, should be performed in the future, to confirm that the methodology efficiently detects Las from different geographical origins.

The combination of LAMP with a LFD amplicon detection system, allows for detection of Las at a speed not previously reported, taking just 45 minutes from the start of the amplification to the evaluation of the results. This characteristic combined with the capability to be carried out in a low resource setting makes the method presented here a powerful diagnostic tool for HLB.

\section{Conclusions}

In this work, we targeted a sequence on the gene CLIBASIA_05175 to develop and validate a LAMP methodology for detection of Las in both host plants and vector insects. To the best of our knowledge, this study constitutes the first report of an isothermal-lateral flow dipstick coupled detection system for diagnosis of HLB with the potential for "in field" applications. This alternative approach was demonstrated to be fast, sensitive and specific in different kinds of samples including leaf material or psyllids. The results of this study provide evidence that this LAMP-based method can be reliably integrated into the HLB management as a tool for faster diagnostics.

\section{Methods}

\section{Biological samples}

Citrus leaf samples were collected from Las symptomatic and asymptomatic sweet orange (Citrus sinensis) trees in orchards from Sao Paulo state, Brazil, during summer and transported at room temperature in a sealed container. The samples were maintained a $4^{\circ} \mathrm{C}$ until they were used for DNA purification, typically 1-2 days after collection. Psyllids were collected and stored submerged in $75 \%$ ethanol until DNA extraction, typically $1-2$ days after collection.

\section{DNA extraction}

Midribs were separated from leaf samples and cut into smaller pieces. DNA was extracted using the Wizard ${ }^{\circ}$ Genomic DNA purification Kit, Promega, Madison, WI, USA, according the manufacturer's instructions and resuspended in $100 \mu \mathrm{L}$ of ultrapure water. The presence of Las in the samples was confirmed by real time PCR as described previously [3]. DNA samples from Diaphorina citri were prepared as follows, a single infected insect 


\section{ATGTATAATACCATAGAAATTACTTGGGGTGGAAATGCTAAACCAAGAATTAACCAGATA \\ ATCGCAGATTTTGTTGCAAAGCGTATTAAGGATTGTTCTAGTGGTTGGGATCGATTTGTT \\ AGTATGGGGATCTTGAAAAGTAATTTTTTAGTGGCAGGAATCATATACCATAACTATTGC \\ CCTATATCTCGTGTCATAGAATTATCAGGTGCATCGGATTGTAAATCATGGCTGAGTCGA \\ $\begin{array}{cc}\text { F3 } & \text { F2 } \\ \text { TCAGTATTAAAGGAAATCTATAGCTATCCTTGGAATCAGTTGTGTTGCCAGGCAGTGATT }\end{array}$ \\ F1 B1C \\ CATCGTATTCCTGACGAAGATTATCCTCAACATCGTATGCTCACTTCCTIGGGGGGAATT \\ LB \\ CGCTATCGTATTCCACGTTTACGAGGAAGGAATGCAGCGGAAAATATATATGTGATTACT \\ $B 3 C$ \\ CATGAAGCTTGGATGCATAATAAAATTAATCGACAATCTTCAGTTCATAAGTCGTTGTAA}

Figure 4 Localization of target sequences used for primer construction. Target sequences used for LAMP primer design are underlined and shaded over the whole sequence of the gene CLIBASIA_05175. Solid lines correspond to F3, F2, F1 B1C, B2C and B3C regions. Dashed line corresponds to loop primers binding regions LFC and LB.

was homogenized by vortexing in presence of $200 \mu \mathrm{L}$ of InstaGene $^{\text {Tx }}$ resin $\left(\mathrm{BIORAD}^{\circ}\right)$, incubated at $56^{\circ} \mathrm{C}$ for 20 minutes to activate the resin chelating groups and then incubated for 8 minutes at $100^{\circ} \mathrm{C}$ in order to destroy cellular structures and release the nucleic acids. Five microliters of this preparation were added to the LasLAMP reaction mix as template.

\section{Computational analysis:}

In order to find a suitable DNA region on the genome of Candidatus Liberibacter asiaticus allowing a specific detection of the microorganism, we manually selected hypothetical protein coding regions from the genome for BLASTn searches [24]. The rationale for this is that hypothetical proteins are open reading frames with low or no homology to known protein coding genes. Sequences showing lower homology with sequences from other organisms were selected.

\section{LAMP reaction}

Oligonucleotide LAMP primers were designed according to the published sequence of the gene CLIBASIA_05175 [GenBank: ACT57606.1], from the Candidatus Liberibacter asiaticus genome. The software Primer Explorer version 4 (Net Laboratory, Tokyo, Japan) was used to target the middle region of the gene (Figure 4), resulting in primers Las-F3, Las-B3, Las-FIP and Las-BIP (Table 4). In addition, a set of two Loop primers, Las-LF and Las-LB was generated for reaction acceleration (Table 4). The Las-LAMP assay was performed using a dry thermal block with a $0.5-\mathrm{mL}$ PCR tube holder. The final LAMP conditions used were as follows, $40 \mathrm{pmol}$ each of primers LasFIP and Las-BIP, 5 pmol each of outer primers Las-F3 and Las-B3, 20 pmol each of loop primers Las-LF and Las-LB, $8 \mathrm{U}$ of Bst DNA polymerase, $4.5 \mathrm{mM} \mathrm{MgSO} 4,1.4 \mathrm{mM}$ of
dNTP mix, $20 \mathrm{mM}$ Tris- $\mathrm{HCl}(\mathrm{pH} 8.8), 10 \mathrm{mM} \mathrm{KCl}$, $10 \mathrm{mM}\left(\mathrm{NH}_{4}\right)_{2} \mathrm{SO}_{4}, 0.1 \%$ Triton X-100 and $1.6 \mathrm{M}$ betaine, in a final volume of $25 \mu \mathrm{L}$ including the template. This reaction mix was incubated at $65^{\circ} \mathrm{C}$ for 30 minutes.

\section{Analysis of LAMP products}

The products of amplification were subjected to electrophoresis at $85 \mathrm{~V}$ for 50 minutes on a $1.5 \%$ agarose gel, followed by ethidium bromide staining. To confirm the specificity of the product some bands were cut and sequenced. The sequences obtained were used as queries to perform BLAST searches [24] in order to confirm identity. Lateral flow dipstick analyses of Las-LAMP products were performed as described previously [20,21]. Briefly, a biotin-labeled FIP primer was used in the Las-LAMP reaction. All other components in the reaction mix remained the same as described above, resulting in biotin-labeled Las-LAMP amplicons. A 5' FITC-labeled DNA probe (5'FITC-CTCAACATCGTATGCTCACTT-3') was designed to hybridize in the region between the Las-FIP and LasBIP primers. Twenty picomol of this probe were added at

\section{Table 4 Sequences of primers used for the Las-LAMP}

\section{assay}

\begin{tabular}{llll}
\hline $\begin{array}{l}\text { Primer } \\
\text { name }\end{array}$ & Type & Sequence (5'-3') & Length \\
\hline Las-F3 & F3 & GCCCTATATCTCGTGTCAT & 19 mer \\
Las-B3 & B3 & ATTCCTTCCTCGTAAACGT & 19 mer \\
Las-FIP & FIP (F1C + F2) $)$ & CACAACTGATTCCAAGGATAGCT- & 44 mer \\
& & ATAATTATCAGGTGCATCGGA & \\
Las-BIP & BIP (B1C + B2) & GCCAGGCAGTGATTCATCGTAG- & 39 mer \\
& & ATAGCGAATCCCCCCA & \\
Las-LF & LF & GATCGACTCAGCCATGATTACAA & 24 mer \\
Las-LB & LB & TGACGAAGATTATCCTCAACATCG & 24 mer \\
\hline
\end{tabular}


the end of the Las-LAMP amplification reaction and incubated at $65^{\circ} \mathrm{C}$ for 10 minutes to allow for hybridization. After the hybridization step, $10 \mu \mathrm{L}$ of this product was mixed with $150 \mu \mathrm{L}$ of the strip assay buffer (Milenia ${ }^{\circ}$ HybriDetect, MGHD1, Milenia Biotec, Germany). Finally the LFD strip was submerged into the mixture, and the results were visualized after 5 minutes.

\section{Sensitivity of LAMP and real time PCR}

In order to estimate the sensitivity of the Las-LAMP assay, purified DNA from a Las infected plant was serially diluted and $1 \mu \mathrm{L}$ aliquots of these dilutions were used as template for Las-LAMP and real time PCR. Las-LAMP reactions were performed as mentioned above, and real time PCR was carried out as described previously [3], in a Step One ${ }^{\text {tm }}$ real time PCR system (Applied Biosystems ${ }^{\circ}$ ).

\section{Additional files}

Additional file 1: Figure S1. Pairwise alignment between CLIBASIA_05175 and related sequences from a BLASTn search. A. BLASTn pairwise alignment between CLIBASIA_05175 (green) and a related sequence from Candidatus Liberibacter solanacearum (black). Las-LAMP primer binding sites are highlighted in yellow and cyan. B. BLASTn pairwise alignment between CLIBASIA_05175 (green) and a related sequence from Candidatus Liberibacter americanus (black). Las-LAMP primer binding sites are highlited in yellow and cyan. C. BLASTn pairwise alignment between CLIBASIA_05175 (green) and a related sequence from Candidatus crescens (black). Las-LAMP primer binding sites are highlighted in yellow and in cyan.

Additional file 2: Figure S2. Evaluation of Candidatus Liberibacter americanus DNA by Las-LAMP. Purified DNA from plants infected with Candidatus Liberibacter asiaticus (Las) or Candidatus Liberibacter americanus (Lam) were used as templates for the Las-LAMP amplification reaction. A. Amplification products analyzed by gel electrophoresis. B. Amplification products analyzed using a lateral flow dipstick. $\mathrm{C}^{-}$: negative control without template. $\mathrm{M}: 1 \mathrm{~Kb}$ plus DNA ladder (Invitrogen ${ }^{\circledast}$ ), the size of the bands is, from bottom to top: $100 \mathrm{bp}$, 200 bp, 300 bp, 400 bp, 500 bp, 650 bp, 850 bp, 1000 bp, 1650 bp, $2000 \mathrm{bp}$ and increments of $1000 \mathrm{bp}$ up to $12000 \mathrm{bp}$.

Additional file 3: Figure S3. Pairwise alignment between CLIBASIA_05175 and the sequence of a Las-LAMP amplification product. A Las-LAMP amplification product band was Analyzed by sequencing. The sequence corresponding to the amplification product (red), from F2 to B2C, has been subjected to a pairwise alignment against CLIBASIA_05175 (green)

Additional file 4: Figure S4. Evaluation of different heating devices on Las-LAMP amplification. A thermal cycler (1), a water bath (2) or an incubator (3), were used to maintain the temperature required for Las-LAMP amplification reaction. A. Amplification products were Analyzed by gel electrophoresis. B. Amplification products Analyzed using a lateral flow dipstick. $\mathrm{C}^{-}$: negative control without Template. $\mathrm{M}: 1 \mathrm{~Kb}$ plus DNA ladder (Invitrogen ${ }^{\circledast}$ ), the size of the bands is from bottom to top: $100 \mathrm{bp}$, 200 bp, 300 bp, 400 bp, 500 bp, 650 bp, 850 bp, 1000 bp, 1650 bp, $2000 \mathrm{bp}$ and increments of $1000 \mathrm{bp}$ up to $12000 \mathrm{bp}$.

Additional file 5: Figure S5. Images of gel electrophoresis and lateral flow dipsticks corresponding to Table 1 and Table 2. A. Images of gel electrophoresis (left) and lateral flow dipsticks (right) corresponding to samples in Table 1. 1. Candidatus Liberibacter asiaticus, 2. Xylella fastidiosa, 3. Xanthomonas campestris pv. campestris, 4. Xanthomonas campestris pv. vesicatoria, 5. Pseudomonas syringae, 6. Botrytis cinerea, 7. Phytophthora citricola, 8. Guignardia citricarpa, 9. Elsinoe fawcettii, 10. Healthy Orange, 11. Healty Citrus limon, 12. Healty Diaphorina citri. B. Images of gel electrophoresis (left) and lateral flow dipsticks (right) corresponding to samples in Table 2. 1. 100 ng DNA, 2. 10 ng DNA, 3. 1 ng DNA, 4. 100 pg
DNA, 5. 10 pg DNA, 6. 1 pg DNA, 7. 100 fg DNA. For all gels, M: 1 Kb plus DNA ladder (Invitrogen ${ }^{\circledast}$ ), the size of the bands is from bottom to top: 100 bp, 200 bp, 300 bp, 400 bp, 500 bp, 650 bp, 850 bp, 1000 bp, $1650 \mathrm{bp}, 2000 \mathrm{bp}$ and increments of $1000 \mathrm{bp}$ up to $12000 \mathrm{bp}$.

\section{Competing interests}

The authors declare that they have no competing interests.

\section{Authors' contributions}

LAR designed the experiments, performed the experimental work and wrote the manuscript; FM performed experimental work and wrote the manuscript, IGO and MPF performed experiments with DNA from Candidatus Liberibacter americanus. MRM, AMDA and APC contributed to coordinate the study and wrote the manuscript; AAV participated in the analysis and interpretation of the data and wrote the manuscript. All authors read and approved the final manuscript.

\section{Acknowledgements}

We thank Dr. Keith Ireton for critical review of the manuscript. We are grateful to Dr. Nelson Arno Wulff of Fundecitrus, São Paulo, Brazil, for providing some of the DNA samples used in this study. Thanks to OPENCLIPART.ORG for providing community sourced images that were used to create illustrations in this manuscript. MRM, APC and AAV are Career Investigators of Consejo Nacional de Investigaciones Científicas y Técnicas (CONICET), Argentina. This work was supported by Agencia de Promoción Científica y Tecnológica of Argentina.

\section{Author details}

${ }^{1}$ Instituto de Ciencia y Tecnología Dr. Cesar Milstein, Fundación Pablo Cassará, Consejo Nacional de Investigaciones Científicas y Técnicas (CONICET), Ciudad de Buenos Aires, Argentina. ${ }^{2}$ Estación Experimental Agroindustrial Obispo Colombres (EEAOC)- CONICET, Instituto de Tecnología Agroindustrial del Noroeste Argentino (ITANOA), Las Talitas, Tucumán, Argentina. ${ }^{3}$ Instituto de Biología Molecular y Celular de Rosario, Departamento de Microbiología, Facultad de Ciencias, Bioquímicas y Farmacéuticas, Universidad Nacional de Rosario, Rosario, Argentina. ${ }^{4}$ Embrapa, Brasília, Distrito Federal, Brasil. ${ }^{5}$ Department of Microbiology and Immunology, University of Otago, Dunedin, Otago, New Zealand. ${ }^{6}$ Instituto de investigaciones fisiológicas y ecológicas vinculadas a la Agricultura (IFEVA-FAUBA), Universidad de Buenos Aires, Ciudad de Buenos Aires, Argentina.

Received: 20 September 2013 Accepted: 26 March 2014 Published: 6 April 2014

\section{References}

1. Gottwald TR: Current epidemiological understanding of citrus Huanglongbing. Annu Rev Phytopathol 2010, 48:119-139.

2. Wang N, Trivedi P: Citrus huanglongbing: a newly relevant disease presents unprecedented challenges. Phytopathology 2013, 103(7):652-665.

3. Li W, Hartung JS, Levy L: Quantitative real-time PCR for detection and identification of Candidatus Liberibacter species associated with citrus huanglongbing. J Microbiol Methods 2006, 66(1):104-115.

4. Morgan JK, Zhou L, Li W, Shatters RG, Keremane M, Duan YP: Improved real-time PCR detection of 'Candidatus Liberibacter asiaticus' from citrus and psyllid hosts by targeting the intragenic tandem-repeats of its prophage genes. Mol Cell Probes 2012, 26(2):90-98.

5. Do Carmo Teixeira D, Luc Danet J, Eveillard S, Cristina Martins E, De Jesus Junior WC, Takao Yamamoto P, Aparecido Lopes S, Beozzo Bassanezi R, Juliano Ayres A, Saillard C, Bove JM: Citrus huanglongbing in Sao Paulo State, Brazil: PCR detection of the 'Candidatus' Liberibacter species associated with the disease. Mol Cell Probes 2005, 19(3):173-179.

6. Grafton-Cardwell EE, Stelinski LL, Stansly PA: Biology and management of Asian citrus psyllid, vector of the huanglongbing pathogens. Annu Rev Entomol 2013, 58:413-432.

7. Manjunath KL, Halbert SE, Ramadugu C, Webb S, Lee RF: Detection of 'Candidatus Liberibacter asiaticus' in Diaphorina citri and its importance in the management of citrus huanglongbing in Florida. Phytopathology 2008, 98(4):387-396.

8. Garnier M, Martin-Gros G, Bove JM: Monoclonal antibodies against the bacterial-like organism associated with citrus greening disease. Ann Inst Pasteur Microbiol 1987, 138(6):639-650. 
9. JM B: Huanglongbing: a destructive, newly emerging, century-old disease of citrus. J Plant Pathol 2006, 88:7-37.

10. Hocquellet A, Bove JM, Garnier M: Production and evaluation of nonradioactive probes for the detection of the two 'Candidatus Liberobacter' species associated with citrus huanglongbing (greening). Mol Cell Probes 1997, 11(6):433-438.

11. Okuda MMM, Tanaka Y: Characterization of the tufB-secE-nusG-rpIKAJLrpoB Gene Cluster of the Citrus Greening Organism and Detection by Loop-Mediated Isothermal Amplification. Plant Dis 2005, 89(7):705-711.

12. Teixeira DC, Saillard C, Couture C, Martins EC, Wulff NA, Eveillard-Jagoueix S, Yamamoto PT, Ayres AJ, Bove JM: Distribution and quantification of Candidatus Liberibacter americanus, agent of huanglongbing disease of citrus in Sao Paulo State, Brasil, in leaves of an affected sweet orange tree as determined by PCR. Mol Cell Probes 2008, 22(3):139-150.

13. Jagoueix S, Bove JM, Garnier M: PCR detection of the two 'Candidatus' Liberobacter species associated with greening disease of citrus. Mol Cell Probes 1996, 10(1):43-50

14. Fujikawa T, Iwanami T: Sensitive and robust detection of citrus greening (huanglongbing) bacterium "Candidatus Liberibacter asiaticus" by DNA amplification with new 16S rDNA-specific primers. Mol Cell Probes 2012, 26(5):194-197.

15. Lin H, Chen C, Doddapaneni H, Duan Y, Civerolo EL, Bai X, Zhao X: A new diagnostic system for ultra-sensitive and specific detection and quantification of Candidatus Liberibacter asiaticus, the bacterium associated with citrus Huanglongbing. J Microbiol Methods 2010, 81(1):17-25.

16. Kim JS, Wang N: Characterization of copy numbers of $16 \mathrm{~S}$ rDNA and $16 \mathrm{~S}$ rRNA of Candidatus Liberibacter asiaticus and the implication in detection in planta using quantitative PCR. BMC Res Notes 2009, 2:37.

17. Notomi T, Okayama H, Masubuchi H, Yonekawa T, Watanabe K, Amino N, Hase T: Loop-mediated isothermal amplification of DNA. Nucleic Acids Res 2000, 28(12):E63.

18. Nagamine K, Hase T, Notomi T: Accelerated reaction by loop-mediated isothermal amplification using loop primers. Mol Cell Probes 2002, 16(3):223-229.

19. Kaneko H, Kawana T, Fukushima E, Suzutani T: Tolerance of loop-mediated isothermal amplification to a culture medium and biological substances. J Biochem Biophys Methods 2007, 70(3):499-501.

20. Kiatpathomchai W, Jaroenram W, Arunrut N, Jitrapakdee S, Flegel TW: Shrimp Taura syndrome virus detection by reverse transcription loopmediated isothermal amplification combined with a lateral flow dipstick. J Virol Methods 2008, 153(2):214-217.

21. Khunthong S, Jaroenram W, Arunrut N, Suebsing R, Mungsantisuk I, Kiatpathomchai W: Rapid and sensitive detection of shrimp yellow head virus by loop-mediated isothermal amplification combined with a lateral flow dipstick. J Virol Methods 2013, 188(1-2):51-56.

22. Rigano LA, Marano MR, Castagnaro AP, Do Amaral AM, Vojnov AA: Rapid and sensitive detection of Citrus Bacterial Canker by loop-mediated isothermal amplification combined with simple visual evaluation methods. BMC Microbiol 2010, 10:176.

23. Duan Y, Zhou L, Hall DG, Li W, Doddapaneni H, Lin H, Liu L, Vahling CM, Gabriel DW, Williams KP, Dickerman A, Sun Y, Gottwald T: Complete genome sequence of citrus huanglongbing bacterium, 'Candidatus Liberibacter asiaticus' obtained through metagenomics. Mol Plant Microbe Interact 2009, 22(8):1011-1020.

24. Altschul SF, Gish W, Miller W, Myers EW, Lipman DJ: Basic local alignment search tool. J Mol Biol 1990, 215(3):403-410.

25. Tindall KR, Kunkel TA: Fidelity of DNA synthesis by the Thermus aquaticus DNA polymerase. Biochemistry 1988, 27(16):6008-6013.

26. LaBarre P, Hawkins KR, Gerlach J, Wilmoth J, Beddoe A, Singleton J, Boyle D, Weigl B: A simple, inexpensive device for nucleic acid amplification without electricity-toward instrument-free molecular diagnostics in low-resource settings. PLoS One 2011, 6(5):e19738.

\section{doi:10.1186/1471-2180-14-86}

Cite this article as: Rigano et al:: Rapid and sensitive detection of Candidatus Liberibacter asiaticus by loop mediated isothermal amplification combined with a lateral flow dipstick. BMC Microbiology 2014 14:86.

\section{Submit your next manuscript to BioMed Central and take full advantage of:}

- Convenient online submission

- Thorough peer review

- No space constraints or color figure charges

- Immediate publication on acceptance

- Inclusion in PubMed, CAS, Scopus and Google Scholar

- Research which is freely available for redistribution

Submit your manuscript at www.biomedcentral.com/submit
() Biomed Central 